\title{
Spectrophotometric analysis of the T Tauri star GQ Lupi A
}

\author{
E. Seperuelo Duarte ${ }^{1,2}$, S. H. P. Alencar ${ }^{3}$, C. Batalha ${ }^{2,4}$, and D. Lopes ${ }^{2}$ \\ ${ }^{1}$ Centro Federal de Educação Tecnológica de Química de Nilópolis, 26530-060 Nilópolis, RJ, Brazil \\ e-mail: esduarte@cefeteq.br \\ 2 Observatório Nacional, Rua General José Cristino 77, São Cristóvão, 20921-400 Rio de Janeiro, RJ, Brazil \\ 3 Departamento de Física - ICEx - UFMG, Av. Antônio Carlos 6627, 30270-901 Belo Horizonte, MG, Brazil \\ ${ }^{4}$ Evergreen Valley College - San Jose, CA 95135, USA
}

Received 13 July 2007 / Accepted 30 June 2008

\section{ABSTRACT}

\begin{abstract}
Context. GQ Lup A is a classical T Tauri star that shows clear signs of accretion through the presence of inverse P Cygni profiles in its main emission lines. Recently, Neuhäuser et al. (2005, A\&A, 435, L13) found a co-moving sub-stellar companion of GQ Lup A, raising the importance of determining precise stellar parameters, system age, and distance to GQ Lup.

Aims. Our main objective is to use spectrophotometric time series to determine GQ Lup A stellar parameters and predict its photospheric spectral distribution. The excess spectral luminosity can then be measured and employed to test predictions of magnetospheric accretion models of classical T Tauri stars.

Methods. We present the analysis of 18 spectrophotometric observations of the T Tauri star GQ Lup A (K7) obtained with the Boller \& Chivens spectrograph at the $1.52 \mathrm{~m}$ ESO telescope in La Silla. We also revisited archival photometric data of this star, comparing previous light curve variability with our more recent data.

Results. We determined the photospheric flux of GQ Lup $\mathrm{A}$ on each observing night and obtained the stellar radius $\left(R_{\star}=\right.$ $1.8 \pm 0.3 R_{\odot}$ ), adopting a mean distance of $150 \pm 20 \mathrm{pc}$ to the Lupus 1 cloud. Assuming a $\mathrm{K} 7 \mathrm{~V}$ temperature of $4060 \mathrm{~K}$, the luminosity of GQ Lup A is $L_{\star}=0.8 \pm 0.3 L_{\odot}$. Standard evolutionary models indicate a stellar mass of $M_{\star}=0.8 \pm 0.2 M_{\odot}$ and an age of $3 \pm 2 \mathrm{Myr}$. GQ Lup A spectral lines are consistent with a projected rotational velocity of $v \sin i=6.5 \pm 2.0 \mathrm{~km} \mathrm{~s}^{-1}$. We measured the excess emission - veiling - and used the resulting photospheric spectral distribution to calculate a stellar extinction $\left(A_{V}\right)$ of $0.5 \pm 0.1$. The veiling was found to be variable and periodic at $10.7 \pm 1.6$ days, which is consistent with the period of GQ Lup A obtained from archival $B$ band photometric data (10.43 \pm 0.12 days). The star exhibits strong emission lines with substantial variability in flux. The emission line fluxes are strongly correlated with one another but not with veiling.
\end{abstract}

Key words. stars: pre-main sequence - stars: activity - techniques: spectroscopic - accretion, accretion disks

\section{Introduction}

T Tauri stars are young, optically visible, low-mass stars. Some of them, known as weak-lined T Tauri stars, do not exhibit evidence of disk accretion, while others, the so-called classical $\mathrm{T}$ Tauri stars (CTTSs) still do. CTTSs present broad emission lines and are spectroscopic and photometrically variable. They show ultraviolet (UV), optical and infrared (IR) excess with respect to the photospheric flux. They have strong magnetic fields $(\sim 2 \mathrm{kG})$ and are X-ray emitters.

Magnetospheric accretion models are the current consensus to explain the main observed characteristics of CTTSs (Shu et al. 1994; Hartmann et al. 1994; Muzerolle et al. 1998, 2001). In these models, the stellar magnetosphere is strong enough to disrupt the circumstellar disk at a few stellar radii. Material at the inner disk, ionized by stellar radiation, is then guided by field lines and collides with the stellar photosphere at near free-fall velocities, creating hot spots at the stellar surface. In this scenario, the permitted emission lines are formed in the accretion funnel and the hot spot emits a continuum flux that veils the photospheric spectrum (Calvet \& Gullbring 1998).

GQ Lup A is a classical T Tauri star with spectral type K7V. It is located in the Lupus molecular cloud complex, near Lupus 1 , at $150 \pm 20$ pc from the Sun (Crawford 2000; Franco 2002). We adopted the distance of Lupus 1 obtained by Franco (2002), following the discussion in McElwain et al. (2007). Recently
Neuhäuser et al. (2008) have measured the parallax of GQ Lup A and $\mathrm{B}$ and found their distances to be $156 \pm 50 \mathrm{pc}$ and $139 \pm 45 \mathrm{pc}$, both consistent with our adopted distance. GQ Lup A presents variable excess continuum flux (veiling) and inverse P Cygni (IPC) profiles (Batalha et al. 2001, hereafter BLB).

The extinction to GQ Lup was estimated to be 0.95 mag in $V$ by Hughes et al. (1994), who also made a first attempt to determine the absolute parameters of the star. Batalha et al. (2001) reported spectrophotometric observations of GQ Lup consisting of 32 medium resolution échelle spectra distributed in two periods of six and eight consecutive nights in 1998. They found variability in the veiling and in the IPC profiles. A correlation between $\mathrm{H} \beta$ IPC and veiling was found, which gives support to the magnetosphere accretion theory, since they both depend on the accretion mass density.

Recently, Neuhäuser et al. (2005) found a co-moving substellar companion to GQ Lup A. The interest has then grown to determine precise parameters, such as age and distance, for GQ Lup A itself. Although GQ Lup A is a good laboratory to test predictions of magnetospheric accretion models due its proximity and relatively large brightness $(V \sim 11.4 \mathrm{mag})$, its astrophysical parameters, such as radius, photospheric luminosity, mass and age are not yet determined with veiling subtraction techniques. In this paper we determine these and other parameters, and compare our results with previous works. In addition, we 
Table 1. Journal of observations.

\begin{tabular}{lll}
\hline \hline Obs. & UT Date and Time & JD-2 400 000.0 \\
\hline 1 & 1999 Feb. 09, 09:14:32 & 51218.885 \\
2 & 1999 Feb. 10, 08:22:14 & 51219.849 \\
3 & 1999 Feb. 11, 08:16:23 & 51220.845 \\
4 & 1999 Feb. 12, 08:15:10 & 51221.844 \\
5 & 1999 Feb. 13, 09:00:01 & 51222.875 \\
6 & 1999 Feb. 14, 08:49:31 & 51223.868 \\
7 & 1999 Feb. 15, 08:33:36 & 51224.857 \\
8 & 1999 Feb. 16, 08:27:56 & 51225.853 \\
9 & 1999 Feb. 17, 08:08:50 & 51226.839 \\
10 & 1999 Feb. 18, 08:33:49 & 51227.857 \\
11 & 1999 Feb. 19, 08:02:06 & 51228.835 \\
12 & 1999 Feb. 20, 07:53:29 & 51229.829 \\
13 & 1999 Feb. 21, 08:31:31 & 51230.855 \\
14 & 1999 Feb. 22, 08:43:46 & 51231.864 \\
15 & 1999 Feb. 23, 08:49:47 & 51232.868 \\
16 & 1999 Feb. 24, 08:54:47 & 51233.871 \\
17 & 1999 Feb. 25, 08:49:19 & 51234.868 \\
18 & 1999 Feb. 26, 09:04:33 & 51235.878 \\
\hline
\end{tabular}

test magnetospheric accretion models comparing our data with general model predictions.

In the following sections we present additional spectrophotometric data of GQ Lup A. We monitor the continuum and spectral line variabilities, improving thereby our knowledge of the circumstellar environment of CTTSs. In Sect. 2 we describe the observations and reduction procedures. The results are presented in Sect. 3 and discussed in Sect. 4. In Sect. 5 we show our conclusions.

\section{Observations}

The observations were carried out in February 1999 with the ESO $1.52 \mathrm{~m}$ telescope at La Silla with the Boller \& Chivens spectrograph. They consist of eighteen exposures listed in Table 1 obtained during eighteen consecutive nights. The first eleven nights were observed with grating No. 26 and a setting that covers the spectral range of $4200 \AA<\lambda<6200 \AA$, while the last seven nights were observed with grating No. 34, covering the spectral range of $3500 \AA<\lambda<7200 \AA$. The spectrograph feeds a Loral Lesser CCD instrument $(2048 \times 2048)$. Gratings chosen (Nos. 26 and 34) have the maximum response at $5730 \AA$ and $3696 \AA$, respectively. The slit opening (4.05") provides an effective resolution of $3.9 \AA$ in the first set and $8.6 \AA$ in the second. In the twelfth night the slit was opened at $4.6^{\prime \prime}$, providing a resolution of $9.6 \AA$ for this exposure only. Two spectrophotometric standards were observed before the target and used as flux calibrators (Hiltner 600 and CD 32-997). Data reduction and spectrophotometric calibrations were performed in a standard way, following the steps outlined in BLB.

In Figs. 1 and 2 we present the reduction and flux calibration results for the two observational sets. We can see continuum and emission lines variability throughout the nights and the lack of inverse P Cygni profiles that were present in BLB.

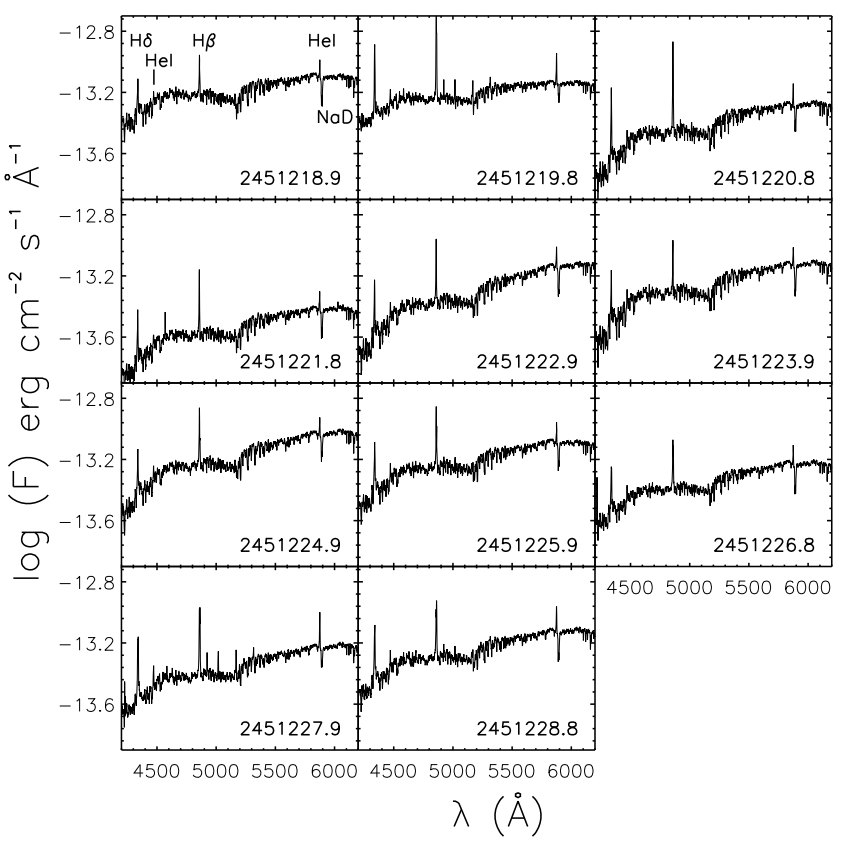

Fig. 1. Spectrophotometric observations of GQ Lup A in February 1999 with grating No. 26. The numbers inside each panel are the Julian Dates of the observations. Some emission lines are identified in the left upper panel.

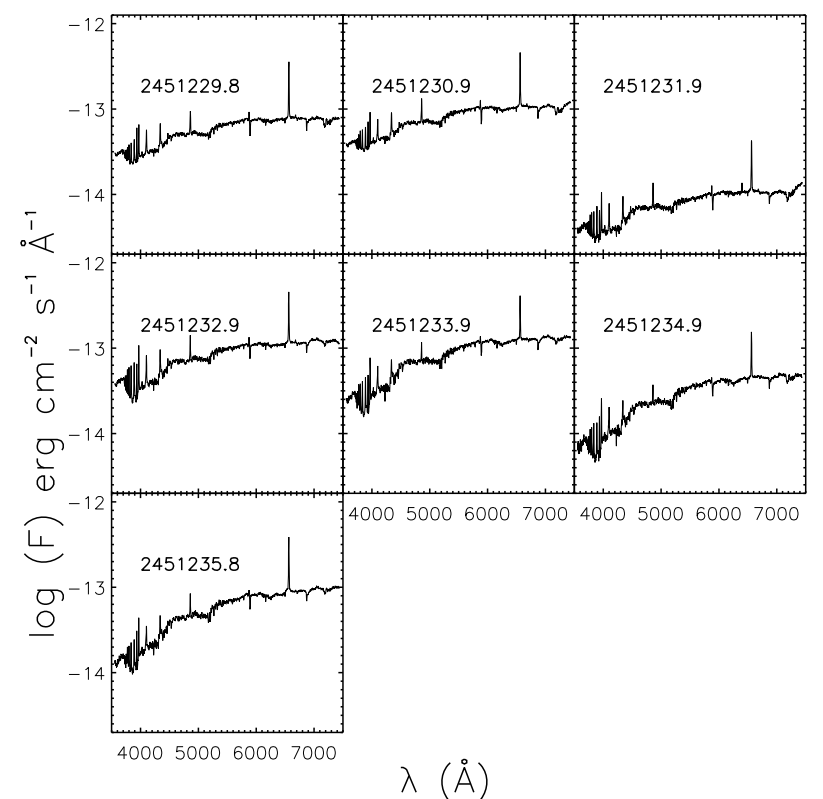

Fig. 2. Spectrophotometric observations of GQ Lup A in February 1999 with grating No. 34. The numbers inside each panel are the Julian Dates of the observations.

\section{Results}

\subsection{Veiling}

Veiling is thought to be produced in hot spots created by the shock of accreting material at the stellar surface. Hot spots have temperatures higher than the photosphere and emit a continuum flux similar to a black body radiation, which peaks at the UV (Calvet \& Gullbring 1998; Gullbring et al. 2000). The observed spectrum is therefore characterized by an excess flux in the blue region and shallower (veiled) photospheric absorption lines than would be expected for their spectral types. 
Table 2. Absorption lines used to measure the veiling and veiling values obtained with each line at each night.

\begin{tabular}{|c|c|c|c|c|c|c|c|c|c|c|c|c|c|c|c|c|c|c|c|}
\hline Lines & $\overline{\lambda \lambda(\AA)}$ & $\bar{~} 1$ & $\overline{2}$ & 3 & 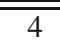 & $\bar{~} \overline{5}$ & $\bar{~} 6$ & 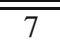 & $\overline{88}$ & $\overline{99}$ & $\bar{~} 10$ & 1811 & 1212 & 213 & 14 & 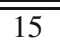 & $\overline{c 16}$ & 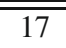 & $\overline{18}$ \\
\hline $\mathrm{FeI}$ & 3550 & & & & & & & & & & & & 5.7 & 4.9 & 3.0 & 4.2 & 2.6 & 1.3 & 2.7 \\
\hline $\mathrm{CaI}$ & 4226 & 1.6 & 3.0 & 0.9 & 1.4 & 1.1 & 0.7 & 1.0 & 1.4 & 1.7 & 2.2 & 1.6 & 2.1 & 2.1 & 1.4 & 1.2 & 0.6 & 0.5 & 0.8 \\
\hline FeI & 4383 & 0.7 & 3.2 & 0.6 & 0.7 & 0.5 & 0.2 & 0.6 & 0.6 & 0.9 & & 1.1 & 1.3 & 1.1 & 0.7 & 0.6 & 0.2 & 0.3 & 0.3 \\
\hline $\mathrm{FeI}$ & 4404 & 0.6 & 1.4 & 0.6 & 0.5 & 0.4 & 0.2 & 0.5 & 0.6 & 0.8 & 1.6 & 0.8 & 0.8 & 1.1 & 0.6 & 0.3 & 0.0 & 0.2 & 0.2 \\
\hline $\mathrm{FeI}$ & 4459 & 0.7 & 1.7 & 0.8 & 0.7 & 0.5 & 0.4 & 0.7 & 1.1 & 1.1 & 1.0 & 1.2 & 1.1 & 1.5 & 0.9 & 0.7 & 0.3 & 0.3 & 0.4 \\
\hline $\mathrm{FeI}$ & 4528 & 0.7 & 1.2 & 0.3 & 0.7 & 0.5 & 0.4 & 0.7 & 0.6 & 0.8 & 0.7 & 0.8 & 1.1 & 1.0 & 0.7 & 0.6 & 0.3 & 0.4 & 0.3 \\
\hline $\mathrm{FeI}$ & 4920 & 0.7 & & 1.0 & 1.0 & 0.7 & 0.4 & 0.6 & 0.5 & 1.0 & & 1.4 & 1.1 & 1.1 & 0.8 & 0.7 & 0.1 & 0.5 & 0.5 \\
\hline $\mathrm{FeI}$ & 4934 & 0.4 & 0.9 & 0.2 & 0.4 & 0.1 & 0.1 & 0.2 & 0.1 & 0.5 & 0.4 & 0.5 & 0.3 & 0.2 & 0.0 & 0.0 & 0.0 & 0.0 & 0.0 \\
\hline $\mathrm{FeI}$ & 4957 & 0.4 & 0.8 & 0.3 & 0.5 & 0.2 & 0.2 & 0.4 & 0.3 & 0.6 & 0.9 & 0.5 & 0.6 & 0.4 & 0.3 & 0.2 & 0.0 & 0.0 & 0.0 \\
\hline FeI & 4982 & 0.3 & 0.8 & 0.1 & 0.3 & 0.2 & 0.1 & 0.4 & 0.2 & 0.4 & 0.5 & 0.3 & 0.1 & 0.0 & 0.0 & 0.0 & 0.0 & 0.0 & 0.0 \\
\hline $\mathrm{FeI}$ & 5041 & 0.2 & 0.5 & 0.0 & 0.2 & 0.1 & 0.0 & 0.3 & 0.2 & 0.4 & 0.6 & 0.2 & 0.1 & 0.1 & 0.0 & 0.0 & 0.0 & 0.0 & 0.0 \\
\hline $\mathrm{FeI}$ & 5269 & 0.5 & 1.2 & 0.5 & 0.5 & 0.6 & 0.4 & 0.5 & 0.6 & 0.7 & 0.9 & 0.6 & 0.7 & 0.8 & 0.5 & 0.5 & 0.3 & 0.3 & 0.3 \\
\hline $\mathrm{FeI}$ & 5302 & 0.2 & 0.7 & 0.1 & 0.3 & 0.2 & 0.2 & 0.2 & 0.2 & 0.3 & 0.5 & 0.2 & 0.1 & 0.3 & 0.1 & 0.2 & 0.0 & 0.0 & 0.0 \\
\hline $\mathrm{FeI}$ & 5328 & 0.3 & 0.9 & 0.2 & 0.4 & 0.3 & 0.3 & 0.3 & 0.3 & 0.5 & 0.7 & 0.5 & 0.3 & 0.6 & 0.5 & 0.4 & 0.2 & 0.2 & 0.2 \\
\hline $\mathrm{FeI}$ & 5371 & 0.4 & 0.9 & 0.4 & 0.5 & 0.4 & 0.4 & 0.4 & 0.4 & 0.6 & 0.7 & 0.6 & 0.2 & 0.4 & 0.1 & 0.3 & 0.0 & 0.1 & 0.3 \\
\hline $\mathrm{FeI}$ & 5586 & 0.4 & 0.8 & 0.4 & 0.3 & 0.3 & 0.3 & 0.4 & 0.4 & 0.6 & 0.6 & 0.6 & 0.1 & 0.4 & 0.3 & 0.3 & 0.0 & 0.1 & 0.1 \\
\hline $\mathrm{FeI}$ & 5615 & 0.3 & 0.4 & 0.2 & 0.2 & 0.4 & 0.2 & 0.2 & 0.1 & 0.2 & 0.3 & 0.4 & 0.0 & 0.0 & 0.4 & 0.0 & 0.0 & 0.0 & 0.0 \\
\hline $\mathrm{CaI}$ & 6102 & 0.2 & 0.1 & 0.0 & 0.1 & 0.1 & 0.3 & 0.1 & 0.1 & 0.2 & 0.2 & 0.2 & 0.0 & 0.1 & 0.2 & 0.1 & 0.1 & 0.0 & 0.1 \\
\hline $\mathrm{CaI}$ & 6122 & 0.6 & 0.8 & 0.5 & 0.5 & 0.6 & 0.6 & 0.5 & 0.5 & 0.7 & 0.9 & 0.6 & 0.2 & 0.3 & 0.4 & 0.4 & 0.2 & 0.2 & 0.4 \\
\hline $\mathrm{FeI}$ & 6136 & 0.1 & 0.3 & 0.0 & 0.0 & 0.2 & 0.1 & 0.0 & 0.0 & 0.2 & 0.2 & 0.1 & 0.0 & 0.0 & 0.0 & 0.0 & 0.0 & 0.0 & 0.0 \\
\hline $\mathrm{CaI}$ & 6162 & 0.5 & 0.8 & 0.4 & 0.5 & 0.5 & 0.5 & 0.5 & 0.6 & 0.6 & 0.7 & 0.6 & 0.6 & 0.8 & 0.6 & 0.5 & 0.4 & 0.6 & 0.5 \\
\hline $\mathrm{FeI}$ & 6254 & & & & & & & & & & & & 0.6 & 0.2 & 0.1 & 0.1 & 0.0 & -0.2 & 0.2 \\
\hline FeI & 6280 & & & & & & & & & & & & 0.0 & 0.0 & & 0.0 & 0.0 & 0.0 & 0.0 \\
\hline $\mathrm{FeI}$ & 6358 & & & & & & & & & & & & 0.0 & 0.1 & 0.0 & 0.1 & 0.0 & 0.0 & 0.2 \\
\hline $\mathrm{FeI}$ & 6494 & & & & & & & & & & & & 0.1 & 0.2 & 0.1 & 0.2 & 0.1 & 0.2 & 0.2 \\
\hline $\mathrm{CaI}$ & 6717 & & & & & & & & & & & & 0.0 & 0.0 & 0.0 & 0.0 & 0.0 & 0.0 & 0.0 \\
\hline $\mathrm{FeI}$ & 6870 & & & & & & & & & & & & 0.1 & 0.0 & 0.1 & 0.2 & 0.1 & 0.1 & 0.2 \\
\hline $\mathrm{FeI}$ & 6978 & & & & & & & & & & & & 0.0 & 0.2 & 0.0 & 0.0 & 0.0 & 0.0 & 0.3 \\
\hline $\mathrm{CrI}$ & 7355 & & & & & & & & & & & & 0.0 & 0.0 & 0.0 & 0.0 & 0.0 & 0.0 & 0.0 \\
\hline
\end{tabular}

Veiling can be measured by comparing the observed photospheric lines of a CTTS with a standard star spectrum of the same spectral type and preferentially of the same luminosity class. The ideal comparison star for veiling determination of GQ Lup A would be a K7 weak lined T Tauri star (same luminosity class as the CTTS) with very low chromospheric activity, so that the photospheric lines are not affected by anything external to the photosphere. This is not always easy to find. However, using a K7V standard does not affect the veiling determination significantly. The dispersion of veiling values calculated with different photospheric lines in the same spectral region is generally larger than the effect of using a main-sequence standard. In our calculations, for example, we have a veiling error of about 0.2 for veiling values below 1 and of about 0.3 for veiling values above 1. To measure the veiling of GQ Lup A we used as standard HD157881 (K7V), which presents $[\mathrm{Fe} / \mathrm{H}]=-0.2 \pm 0.2$ (Cayrel de Strobel et al. 2001). The standard spectra were taken from the Stellar Library for Stellar Population Synthesis Models (STELIB) (Le Borgne et al. 2003) and downgraded to our spectral resolution. Veiling was measured according to the method described by Gullbring et al. (1998). We selected 29 strong blends of absorption lines visible in GQ Lup A and standard spectra. The veiling was then obtained according to the relation

$v=\frac{F_{1}^{\mathrm{o}} / F_{\mathrm{c}}^{\mathrm{o}}-F_{1}^{\mathrm{p}} / F_{\mathrm{c}}^{\mathrm{p}}}{1-F_{1}^{\mathrm{o}} / F_{\mathrm{c}}^{\mathrm{o}}}$

where $F_{1}^{\mathrm{o}}$ and $F_{\mathrm{c}}^{\mathrm{o}}$ are the line and continuum observed fluxes, and $F_{1}^{\mathrm{p}}$ and $F_{\mathrm{c}}^{\mathrm{p}}$ are the line and continuum photospheric fluxes.
The spectral lines used in the veiling calculations are listed in Table 2, together with veiling values obtained for each line. The veiling distributions are shown in Fig. 3, indicating variability in the spectral distribution as well as in the absolute veilings at each wavelength. In Fig. 4 we show the changes in veiling as a function of days at two specific wavelengths, $4405 \AA$ (top) and $5586 \AA$ (bottom). As expected, the absolute veiling values as well as variability range are larger in the $B$ band than in the $V$ band. In addition, we note a cyclic behavior in the veiling, especially in the $B$ band. We performed a periodogram analysis of the $B$ band veiling obtaining a period of $10.7 \pm 1.6$ days with a false alarm probability (FAP) of $1.4 \%$. This result is consistent within less than $2 \sigma$ with the period of $8.45 \pm 0.2$ days obtained by Broeg et al. (2007) for GQ Lup A. We used the Scargle (1982) periodogram estimator as modified by Horne \& Baliunas (1986) to handle irregularly spaced data and the implementation of the Cleanest method within the Peranso software (Vanmunster 2007) to calculate the periodograms. The Cleanest method calculates the power spectrum of unequally-spaced data using an advanced implementation of the date compensated discrete Fourier transform, as described by Foster (1995). The FAPs were calculated with the Monte-Carlo method described in Herbst et al. (2002).

\subsection{Photometric periodicities}

We searched for periodicities in the $B$ band photometric data of GQ Lup A reported in the literature (Schwartz \& Noah 1978; 


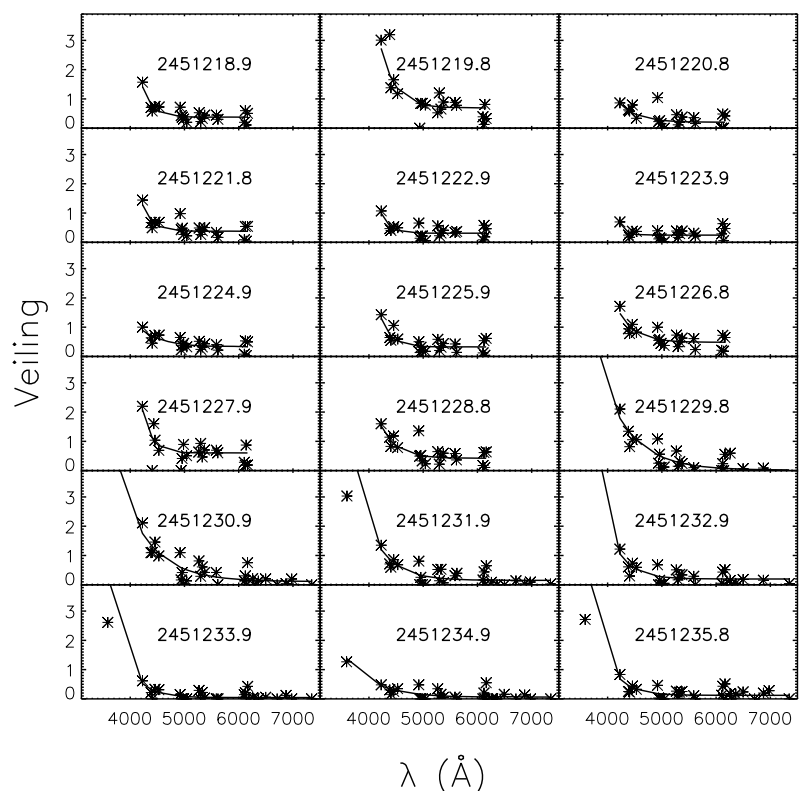

Fig. 3. Calculated veiling values as a function of wavelength. The numbers inside each panel are the Julian Dates of the observations.

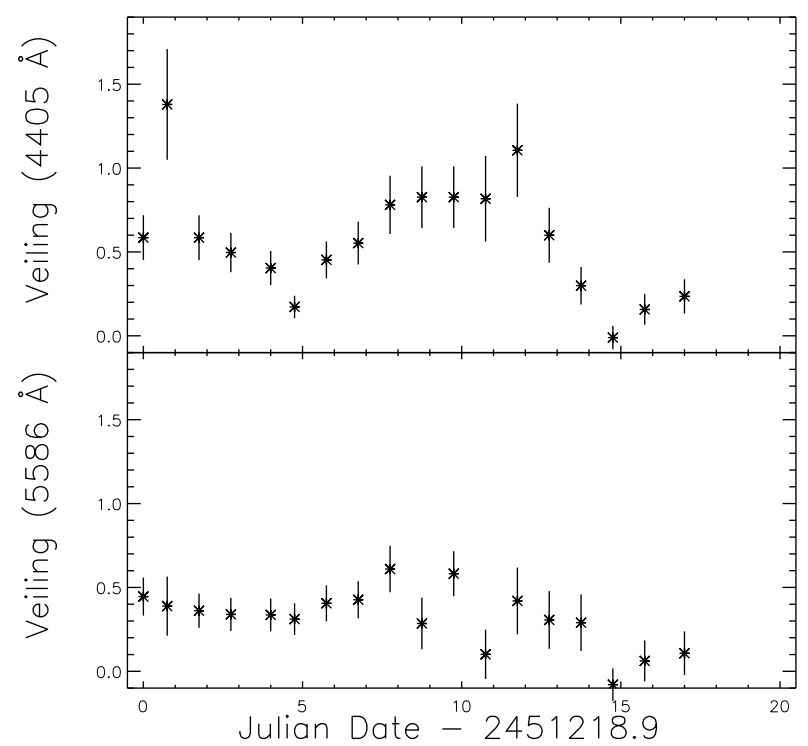

Fig. 4. Calculated veiling values with error bars as a function of days at $4405 \AA$ (top) and $5586 \AA$ (bottom).

Appenzeller et al. 1978; Mundt \& Bastian 1980; Appenzeller et al. 1980; Herbst 1984; Covino et al. 1992). These data are not equally spaced, so we always checked with the Peranso software (Vanmunster 2007) if the periods found were not due to the irregular observational window used. Using all the $B$ band photometric data together, which cover a total of 38 observing nights spanning over several years, we obtained a main period value at $10.43 \pm 0.12$ days but with a very high FAP. The period error comes from the comparision of the several periods obtained with the $B$ and $V$ photometric data using different period search methods (Scargle, Cleanest) and softwares. Periods around 13.73 days were also found with significant power. However, several periods around 13 and 14 days can be aliases caused by the observing rate, so the 13.73 day period cannot be considered as trustful. The archive photometric data has several datasets separated by years and this is problematic for the

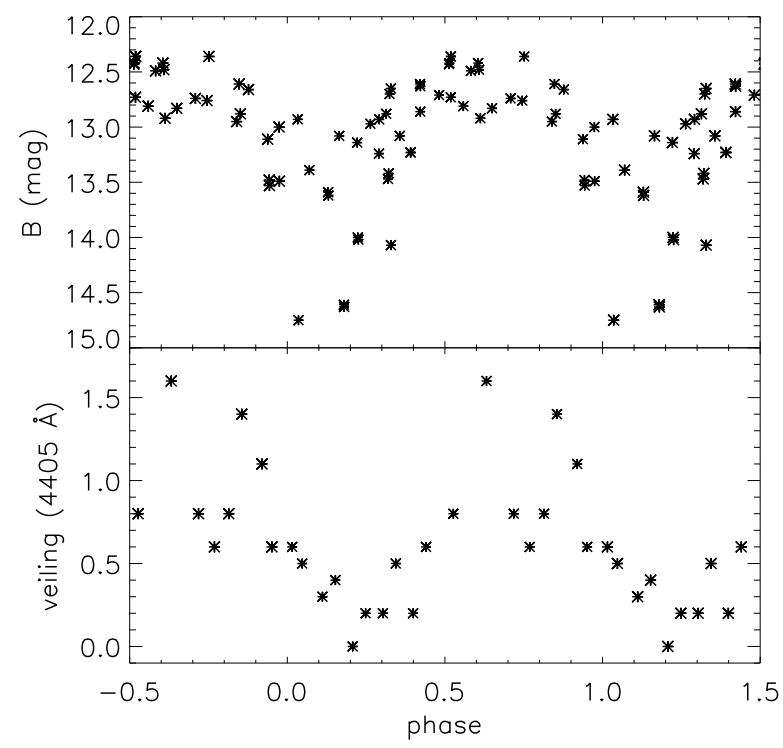

Fig. 5. $B$ band light curve (top) and veiling at $4405 \AA$ (bottom) in phase with a period of 10.43 days (see Sect. 3.2). The $B$ band data come from Schwartz \& Noah (1978), Appenzeller et al. (1978), Mundt \& Bastian (1980), Appenzeller et al. (1980), Herbst (1984), and Covino et al. (1992). The phases were calculated relative to an arbitrary date $(\mathrm{JD}=2441200.0)$.

period search algorithms, since it can lead them to wrong period values. However, since the photometric period agrees with the period obtained with the veiling data, we decided to keep it as a possible period to GQ Lup A.

The light curve of the $B$ band and the veiling are shown in phase with the above period in Fig. 5. The $B$ light curve is characterized by a nearly constant level of maximum brightness ( $B \sim 12.6$ ) with luminosity drops reaching up to 2 mag. We did not attempt to look for periodicities using single datasets, since the single dataset with the largest number of photometric observations is that of Covino et al. (1992), which has 13 observations taken in 7 non-consecutive nights, covering 13 days from the first to the last observation. Even this dataset is not well suited to look for periodic variations of about 10 days. The corresponding $V$ band light curve shows periodicities at 13.73 days and 10.43 days. The 10.43 day period also presents a very high FAP and less power than the periods calculated in the $B$ band. The $V$ band may show variations not only due to veiling changes but also due to changes in the $\mathrm{H} \alpha$ line of GQ Lup A that is known to present strong variability (H $\alpha$ equivalent width of $2.8 \AA$, Herbig \& Bell 1988; and $38.6 \AA$, Hughes et al. 1994) and may originate in different physical regions (accretion funnel flows, winds). It may therefore be more difficult to detect periodicities in the $V$ than in the $B$ band. Although the $B-V$ color is sometimes bluer near the light curve minimum, there is no clear evidence of a strong correlation between $B-V$ and $B$ magnitude (linear correlation coefficient of only 0.6). If the observed $B$ band variability were due to spots (hot or cool), we would expect to see a strong correlation between $B$ and $B-V$, since the brightness variations would correspond to temperature variations at the stellar surface. On the other hand, if the light curve variability were mostly due to occultations by opaque material (e.g. a magnetically-warped inner disk rim), we would not expect to see any correlation between $B$ and $B-V$ and almost no $B-V$ variation. What we see in the GQ Lup A photometric data is an intermediate situation 
where there is a weak correlation between $B$ and $B-V$ and some $B-V$ variability (up to $0.5 \mathrm{mag}$ ). So we are probably seeing the influence of hot and cold spots on the light curve and maybe also some occultation due to opaque material from the accretion disk.

We also looked for periodicities in the GQ Lup data obtained by the All Sky Automated Survey (ASAS) project (Pojmanski 2002). They consist of 4 datasets with 3, 156, 219 and 4 observations each in the $V$ band. We used only the best quality observations (labeled as "grade A") in the second and third datasets containing most of the data and obtained two main periods at 8.31 and 9.47 days, both corresponding very closely to aliases, a false peak caused by the observing rate. We obtained two main periods at 6.22 and 13.16 days with the third data set. The first determination corresponds to an alias due to the observing rate, while the second period can be considered as reliable, although with a lower power. Unfortunately, all the period determinations with the ASAS data presented a very high FAP and the folded light curves are not convincing at all.

Recently Broeg et al. (2007) presented a rotational period determination for GQ Lup A based on new simultaneous optical and near-IR photometric data. They obtained $8.45 \pm 0.2$ days with a combined FAP of $1.5 \%$. Our veiling data folded at that period is considerably worse than that at 10.43 days, our proposed period. The archive $B$-band light curve shows a large amount of scatter when folded at 8.45 days, but given the period error we would only expect the phase to be conserved for about one year, while several datasets in the archive $B$-band photometry are separated by more than that. We will further discuss the different period values and their implications in Sect. 5.

\subsection{Stellar extinction}

We assumed that the interstellar and circumstellar extinction laws are equivalent and computed the GQ Lup A extinction using the method developed by Gullbring et al. (1998). We adjusted the following linear function

$2.5 \log \left[\frac{F_{\lambda}^{\mathrm{s}}}{F_{\lambda}^{\mathrm{o}}}\left(1+v_{\lambda}\right)\right]=-2.5 \log C_{1}+\left(A_{\mathrm{V}}^{\mathrm{o}}-A_{\mathrm{V}}^{\mathrm{s}}\right) \omega_{\lambda}$

where $F_{\lambda}^{\mathrm{s}}$ and $F_{\lambda}^{\mathrm{o}}$ are the standard and GQ Lup A fluxes respectively, $v$ is the veiling, $C_{1}$ is a constant that takes into account the different solid angles subtended by GQ Lup and the template star, $A_{\mathrm{V}}^{\mathrm{o}}$ and $A_{\mathrm{V}}^{\mathrm{s}}$ are the stellar extinctions of GQ Lup $\mathrm{A}$ and the standard, respectively, and $\omega_{\lambda}=A_{\lambda} / A_{\mathrm{V}}$ is the interstellar extinction law. Fitting this function and knowing that $A_{\mathrm{V}}^{\mathrm{s}}=0$ (Le Borgne et al. 2003), we obtain an extinction value of $A_{\mathrm{V}}^{\mathrm{O}}=0.5 \pm 0.1$ mag for GQ Lup A, which is in agreement with the value of $A_{\mathrm{V}}^{\mathrm{o}}=0.4 \pm 0.2$ found by BLB.

\subsection{Physical parameters}

\subsubsection{Photospheric flux}

The photospheric flux is given by

$F_{\lambda}^{\mathrm{p}}=\frac{F_{\lambda}^{\mathrm{o}}}{\left(v_{\lambda}+1\right)} 10^{-0.4 A_{\lambda}^{\mathrm{o}}}$

where $F_{\lambda}^{\mathrm{o}}$ is the observed flux and $A_{\lambda}^{\mathrm{o}}=\omega_{\lambda} A_{\mathrm{V}}^{\mathrm{o}}$. Using the measured veiling values (Sect. 3.1) and $A_{\mathrm{V}}=0.5$ (Sect. 3.3), we calculated the photospheric flux of each observing night, except for 2451231.9 and 2451234.9 , excluded due to the non photometric conditions. The GQ Lup A photosphere was then computed as the mean photospheric flux and is shown in Fig. 6.

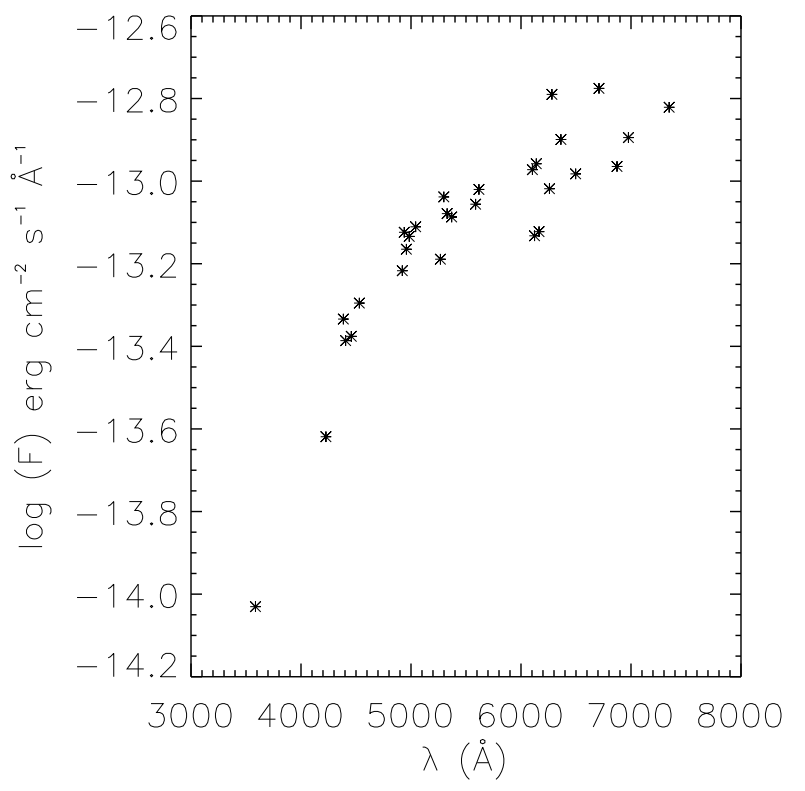

Fig. 6. GQ Lup A photospheric flux.

\subsubsection{Luminosity}

Before computing the GQ Lup A luminosity, we estimate its radius with the following equation

$F(R)=F(d)\left(\frac{d}{R}\right)^{2}$

where $F(R)$ and $F(d)$ are the intrinsic and observed bolometric fluxes respectively, $d$ is the distance to GQ Lup A and $R$ is the stellar radius. However, we only have observations in the optical, so we can not directly obtain, from our data, the observed bolometric flux of GQ Lup A. So first we used photometric data from the K7V standard HD157881 in the literature to calculate its intrinsic flux, assuming a K7V radius of $0.6 R_{\odot}$ (Schmidt-Kaler 1982) and a distance of 7.7 pc (Perryman et al. 1997). We then calculated the standard observed flux at a distance of $150 \pm 20 \mathrm{pc}$, which is the distance to the Lupus 1 cloud where GQ Lup is located (Crawford 2000; Franco 2002), and compared this flux distribution and the GQ Lup A observed flux (Fig. 7, top panel). We varied the standard radius until a good match was found between the two distributions, determining a radius of $1.8 \pm 0.3 R_{\odot}$ for GQ Lup A (Fig. 7 bottom panel). With this value and assuming a K7V temperature of $4060 \mathrm{~K}$ (Schmidt-Kaler 1982), the luminosity of GQ Lup A is $L=0.8 \pm 0.3 L_{\odot}$. Janson et al. (2006) find for GQ Lup A $L=0.5-1.71 L_{\odot}$, which is in agreement with our determined value.

\subsubsection{Mass and age}

Mass and age determination in the Pre-Main Sequence (PMS) is still uncertain due to the difficulties in modelling convection, rotation and accretion and in setting reasonable initial conditions for the PMS (see a review on the subject by Siess 2001). However, the (yet few) mass determinations obtained from young binary systems show that, with all the simplifications actually used in the models and the difficulties to obtain stellar parameters with small errors, the available theoretical models are still able on average to predict stellar masses to within 20\% (Mathieu et al. 2007), which is reasonable.

We placed the measured effective temperature and stellar luminosity of GQ Lup A on evolutionary PMS models of 


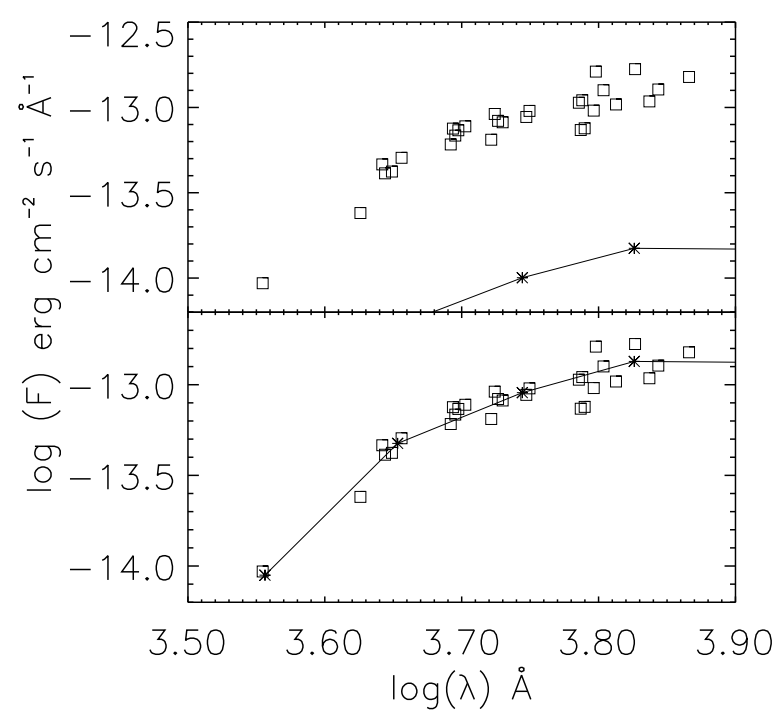

Fig.7. Comparison between GQ Lup A photospheric flux (open squares) and HD 157881 (stars and solid lines). Top panel, supposing HD157881 has $R_{\star}=0.6 R_{\odot}$. Bottom panel, supposing HD 157881 has $R_{\star}=1.8 R_{\odot}$.

Siess et al. (2000) ( $\alpha=1.6)$, Baraffe et al. (1998) $(\alpha=1.5)$, and a modified version of the D'Antona \& Mazzitelli (1997) models $(\alpha=1.5)$ that includes non-grey atmospheres (Landin et al. 2006) (see Fig. 8). Our luminosity and $T_{\text {eff }}$ values indicate a stellar mass of $0.8 \pm 0.2 M_{\odot}$ and an age of $3 \pm 2 \mathrm{Myr}$, taking into account all three models. Janson et al. (2006) obtained ages from 0.7 Myr up to $5 \mathrm{Myr}$ for GQ Lup A, using their determined values of temperature $\left(T_{\text {eff }}=4074 \pm 100 \mathrm{~K}\right)$ and luminosity $\left(0.50 L_{\odot}<L<1.71 L_{\odot}\right)$ and the models of Siess et al. (2000). Recently McElwain et al. (2007) determined an age of $\approx 3 \mathrm{Myr}$ for GQ Lup A with a possible age range of 1-10 Myr, comparing the color magnitude diagram of GQ Lup A $\left(M_{I}\right.$ vs. $\left.R-I\right)$ with evolutionary tracks from Baraffe et al. (1998). The results by Janson et al. (2006) and McElwain et al. (2007) agree with ours and point to ages marginally higher than that of $1 \pm 1 \mathrm{Myr}$ proposed by Neuhäuser et al. (2005).

\subsubsection{Star-disk system inclination}

The projected rotational velocity $(v \sin i)$ of GQ Lup A was obtained by comparing high resolution spectra of GQ Lup A with that of a template star of similar spectral type. High resolution data were obtained in 2000 with the FEROS spectrograph at the ESO $1.52 \mathrm{~m}$ telescope in La Silla. The FEROS spectrum of a $\mathrm{K} 7 \mathrm{~V}$ standard was taken from the SACY catalog of Torres et al. (2006) that had its $v \sin i$ previously determined (HIP 116384 , $\left.v \sin i=4.5 \mathrm{~km} \mathrm{~s}^{-1}\right)$. We applied the veiling value measured in the GQ Lup A spectrum $(v=1.0)$ and rotationally broadened the standard spectrum until a good match with the GQ Lup A spectrum was found. The best match is shown in Fig. 9 and corresponds to $v \sin i=6.5 \pm 2.0 \mathrm{~km} \mathrm{~s}^{-1}$, which is consistent with the value of $v \sin i=6.8 \pm 0.4 \mathrm{~km} \mathrm{~s}^{-1}$ obtained by Guenther et al. (2005) using HARPS spectra. Using the $v \sin i$ value measured by Guenther et al. (2005) due to its higher precision, the stellar radius of $R_{\star}=1.8 \pm 0.3 R_{\odot}$ obtained in Sect. 3.4.2 and the rotational period of $10.43 \pm 0.12$ days obtained with all the available $B$ band photometry, which is consistent with the one

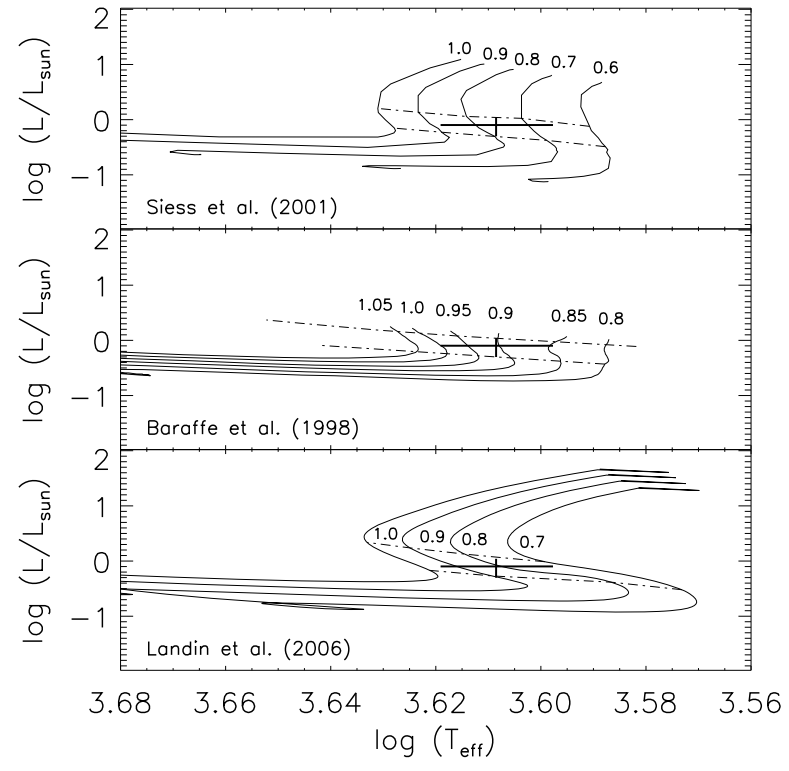

Fig. 8. GQ Lup A luminosity and effective temperature with error bars are shown in the three panels (thick solid lines) overplotted with evolutionary models (thin solid lines) and isochrones (dash-dotted lines). The numbers near each evolutionary track represent the stellar mass in solar masses. Top panel: models by Siess et al. (2000). The isochrones correspond to $1.6 \mathrm{Myr}$ and $5 \mathrm{Myr}$. Middle panel: models by Baraffe et al. (1998). The isochrones correspond to $1.42 \mathrm{Myr}$ and 5.63 Myr. Bottom panel: models by Landin et al. (2006). The isochrones correspond to 1.26 Myr and 5.10 Myr.

measured with our veiling values (see Sects. 3.1 and 3.2), we obtain an inclination angle of $51^{\circ} \pm 13^{\circ}$ for the system. If we use the period obtained from the veiling data, $10.7 \pm 1.6$ days, and the same values of $v \sin i$ and stellar radius, we obtain an inclination of $53^{\circ} \pm 18^{\circ}$ for the system, which is basically the same as the one obtained with the $B$ band period. If we use instead the period determined by Broeg et al. (2007), $8.45 \pm 0.2$ days, the $v \sin i$ value measured by Guenther et al. (2005), and our calculated stellar radius, we obtain an inclination of $39^{\circ} \pm 12^{\circ}$ for the system. Broeg et al. (2007) calculated an inclination of $27^{\circ} \pm 5^{\circ}$ for GQ Lup A, using the same value of $v \sin i$, but a different radius for the star.

\section{Emission lines}

GQ Lup A was observed during 18 nights, the first 11 nights with a mean resolution of $3.9 \AA$ from $4200 \AA$ to $6200 \AA$ and 7 nights with a lower resolution $(8.6 \AA)$, covering the wavelength range from $3550 \AA$ to $7200 \AA$. Lines such as $\mathrm{H} \beta$, He I (5876 $)$ and $\mathrm{NaD}$ where therefore obtained during all the nights, but $\mathrm{H} \alpha$ and $\mathrm{Ca}$ II $\mathrm{H} \& \mathrm{~K}$ were only included in the last 7 nights of observations with the lower resolution setup. GQ Lup A is known for showing inverse P Cygni profiles in the upper Balmer lines (Bertout et al. 1982; Batalha et al. 2001), which are produced when photons from the accretion shock are absorbed by the accreting material falling along magnetic field lines. Curiously, the Balmer lines did not show any inverse P Cygni profiles in our observations. $\mathrm{H} \beta$, instead, presented a double peaked profile with a central or blueshifted absorption component that is likely to be due to winds and not to accretion. Our measured veiling values are lower than those found by BLB in the $B$ band region - maximum veiling value of 1.5 in the present work instead of $\sim 4.4$ in 


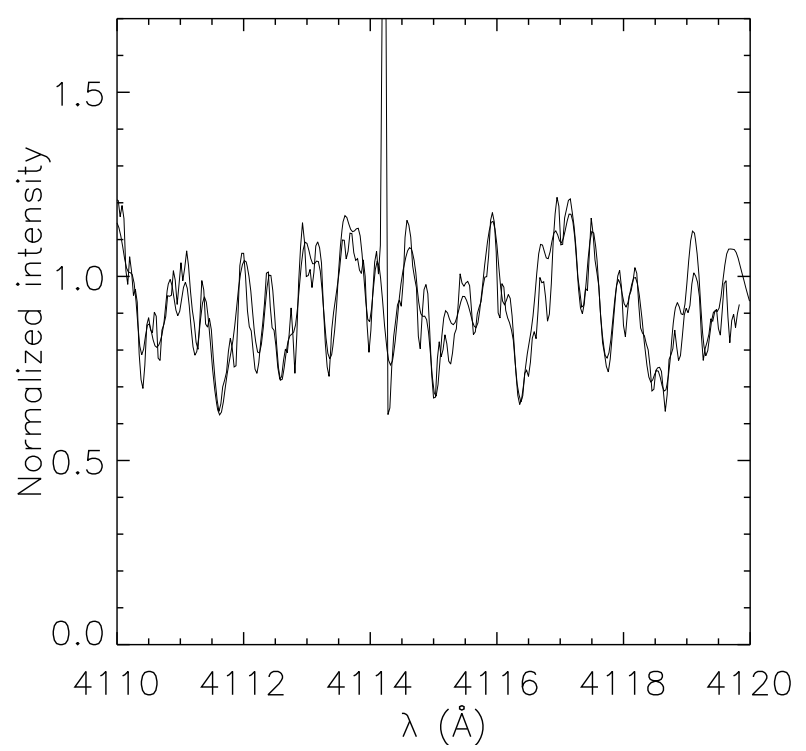

Fig. 9. Comparison between FEROS spectra of GQ Lup A and a K7V standard. The standard spectrum was veiled and rotationally broadened to $6.5 \mathrm{~km} \mathrm{~s}^{-1}$.

BLB. This provides circumstantial evidence that IPC profiles are associated with special viewing angles, which is a fixed parameter for the star-disk system, and episodes of large accretion rates. Redshifted absorption components are not detected in our spectral lines overlying our modest to low continuum veiling. All the emission lines presented variability in intensity and profile shape, but no periodicity was found.

According to magnetospheric accretion models, the emission lines are mainly produced in the accretion funnel (Muzerolle et al. 2001; Kurosawa et al. 2006) and they depend, among other parameters, on the mass accretion rate, as the veiling also does. Some correlation between the emission lines flux and the veiling might then be expected. Muzerolle et al. (1998) found some tendency for the emission lines equivalent width to increase with veiling for low veiling values $(v<2)$, but at high veilings the correlation saturated. Correlations between the Balmer lines and veiling are however not always observed (Johns-Krull \& Basri 1997; Batalha et al. 2002; Petrov et al. 2001).

We measured the total flux of the main emission lines in our spectra and found correlations between hydrogen, calcium and helium lines (Figs. 10-12), as also observed by Alencar \& Basri (2000) and Batalha et al. (2002). This indicates that the main emission lines are at least partly formed in a common region, like the accretion funnel. Like the results presented by BLB, we did not find any correlation between the emission lines total flux and the measured veiling values. BLB found instead a correlation between veiling and redshifted absorption component, the higher the veiling, the stronger the inverse P Cygni component. They argue that veiling variations and the formation of redshifted absorption mostly depend on the mass accretion rate and field configuration near the accretion shock region. Their data and ours seem to indicate that in the present case the emission line flux is ignoring the variations that are at work in the accretion shock region. The emission line flux seems to depend basically on the properties of the accreting gas, like the local gas density, temperature and velocity field, along the accretion funnel.

We cannot directly measure the mass accretion rate of GQ Lup A with our data, but it can be estimated by comparing the observed $\mathrm{H} \beta$ and $\mathrm{NaD}$ lines with magnetospheric accretion profiles from Muzerolle et al. (2001). We did not attempt to obtain

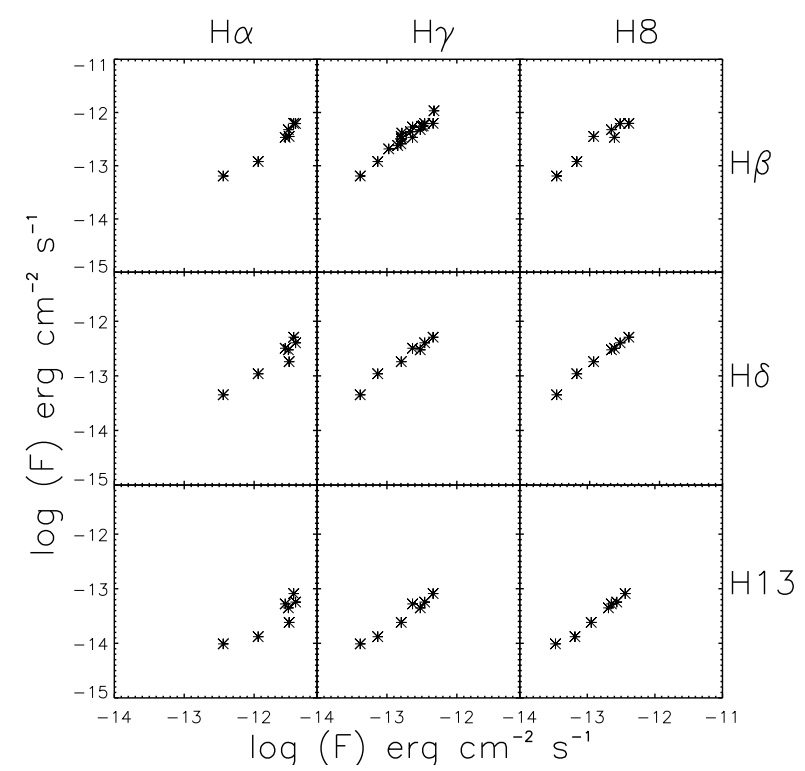

Fig. 10. Correlation between hydrogen lines.

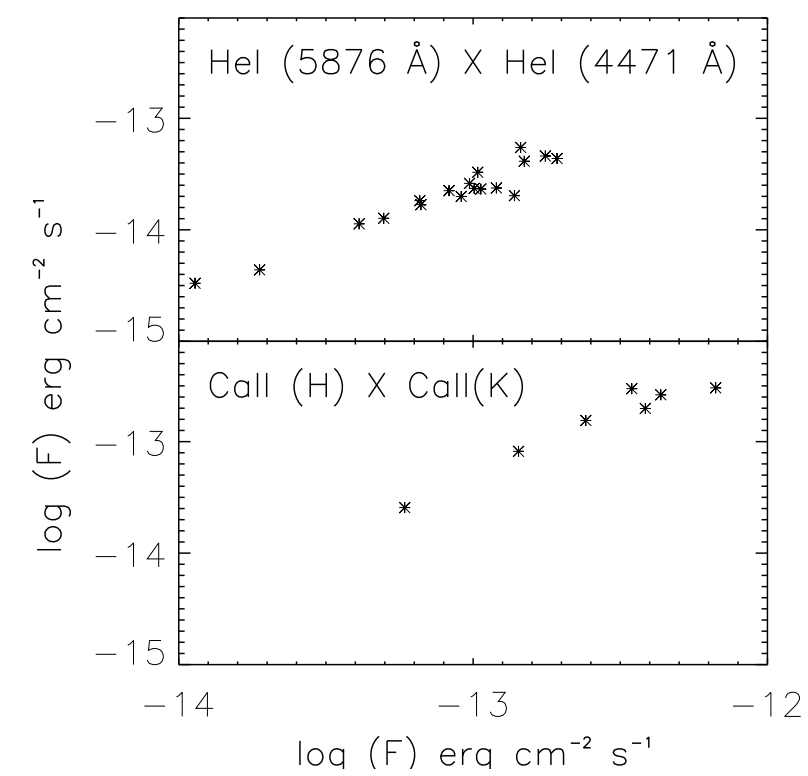

Fig. 11. Correlation between helium lines (top) and calcium lines (bottom).

a perfect quantitative match, but we looked for magnetospheric accretion profiles that presented the same general characteristics as the observed ones. Our observed $\mathrm{H} \beta$ profiles show a blueward asymmetric emission component whose normalized intensity varies from approximately 1.8 to 4 . We do not see any redshifted absorption, but it was often observed by other authors (Bertout et al. 1982; Batalha et al. 2001), so we cannot discard models with IPC profiles. The NaD lines in our spectra are always seen in absorption with intensities that vary from 0.2 to 0.35 . We looked for models calculated with an inclination of $60^{\circ}$ to the line of sight that fall within our estimated inclination of GQ Lup A (see Sect. 3.4.4). The magnetospheric accretion models of Muzerolle et al. (2001) that best simultaneously match the characteristics of both lines described above present a magnetosphere with a small extent and wide width in which the accreting material comes from field lines crossing the disk within 2.2 to $3 R_{\star}$. With these models, we obtain a good match with the 


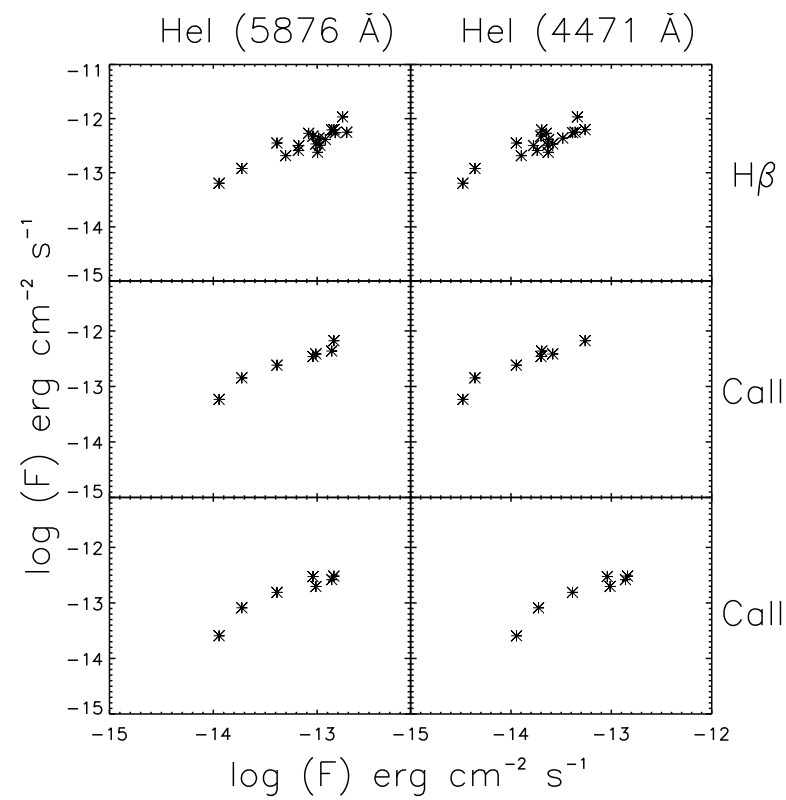

Fig. 12. Correlation between helium, hydrogen and calcium lines.

Table 3. GQ Lup A physical parameters.

\begin{tabular}{ll}
\hline \hline Parameter & This work \\
\hline$A_{V}$ & $0.5 \pm 0.1$ \\
$R\left(R_{\odot}\right)$ & $1.8 \pm 0.3$ \\
$L\left(L_{\odot}\right)$ & $0.8 \pm 0.3$ \\
$M\left(M_{\odot}\right)$ & $0.8 \pm 0.2$ \\
Age $(\mathrm{Myr})$ & $3 \pm 2$ \\
$P($ days $)$ & $10.43 \pm 0.12$ \\
$v \sin i\left(\mathrm{~km} \mathrm{~s}^{-1}\right)$ & $6.5 \pm 2.0$ \\
$i$ & $51^{\circ} \pm 13^{\circ}$ \\
$\dot{M}_{\text {acc }}\left(M_{\odot} \mathrm{yr}^{-1}\right)$ & $10^{-7}$ to $10^{-8}$ \\
\hline
\end{tabular}

following parameters, $\dot{M}_{\text {acc }}=10^{-7} M_{\odot} \mathrm{yr}^{-1}$ and a maximum funnel temperature of $T_{\mathrm{m}}=7000 \mathrm{~K}$ and with $\dot{M}_{\mathrm{acc}}=10^{-8} M_{\odot} \mathrm{yr}^{-1}$ and $T_{\mathrm{m}}=8000 \mathrm{~K}$ or $10000 \mathrm{~K}$. The high veiling values that are often measured in the spectra of GQ Lup A support the idea of a considerable mass accretion rate in the system.

\section{Discussion}

The physical parameters obtained for GQ Lup A are summarized in Table 3. They were calculated assuming that GQ Lup A is located in the Lupus 1 molecular cloud complex, which is at a distance of $150 \pm 20 \mathrm{pc}$ (Crawford 2000; Franco 2002). Some of the parameters in Table 3 were recently obtained by Janson et al. (2006) and McElwain et al. (2007). Our values agree with theirs within our estimated errors.

GQ Lup A is a CTTS that presents variable line fluxes and veiling. The observed periodic veiling variability can be explained by a magnetospheric accretion model with an inclined dipole field (Bertout et al. 1988). The magnetic field inclination with respect to the rotation axis leads to an azimuthal asymmetry in the field lines, which favors accretion where the magnetic axis is closer to the disk plane. In this case, the hot accretion ring is asymmetric and brighter where accretion is favored, leading to a veiling modulation as the system rotates (Mahdavi \& Kenyon 1998; Romanova et al. 2004). Due to that we considered the period obtained from $B$ band photometric data (10.43 \pm 0.12 days, see Sect. 3.2) the rotational period of the star, since it is consistent with the period of $10.7 \pm 1.6$ days obtained from the veiling analysis given the much poorer sampling of the veiling data. In Fig. 5 we show the $B$ light curve and the veiling in phase. The system is in general brighter when the veiling is higher, suggesting that veiling changes contribute to the photometric variability. However the photometric and spectroscopic data were not acquired simultaneously, and the accuracy with which we derived the period at each epoch is not enough to ensure that the phase information was preserved between observations taken at least seven years apart.

The above characteristics can be compared to the results of the simultaneous photometric and spectroscopic campaigns of AA Tau (Bouvier et al. 2003, 2007), another CTTS that presents periodic veiling and light curve variability. While the AA Tau veiling was rather low (from 0.1 to 0.8 ) and showed a strong peak in the deep minimum cycles, near the minimum, the GQ Lup A veiling tends to be higher and seems to peak at maximum brightness. The AA Tau photometric variability was attributed mainly to occultations of the star by a magnetically-warped inner disk edge and not to veiling variations. In that case, the photometric variability occurs almost without any color change except for some $B-V$ brightening events. The AA Tau system presents a high inclination to the line of sight $\left(\sim 75^{\circ}\right)$, which favors occultations by the inner disk, while the lower inclination of GQ Lup $A\left(51^{\circ} \pm 13^{\circ}\right)$ may prevent significant occultations. However we cannot discard that part of the variability observed in GQ Lup A could be due to opaque material, since, like AA Tau, it does not present a strong $B-V$ variability (most of the time $1.0 \mathrm{mag}<B-V<1.2 \mathrm{mag}$, but $B-V$ can reach up to $1.4 \mathrm{mag}$ ). The system also shows a very strong brightness variation (up to $2 \mathrm{mag}$ ) that can hardly be attributed to veiling alone, as discussed later in this Section. Another evidence that obscuration by opaque material contributes to the light curve variability of GQ Lup A comes from the fact that the system becomes bluer when fainter in the deep minima $(V>12)$, both $B-V$ and $U-V$ decreasing for $V<12$ and then increasing for $V>12$. This blueing phenomenon is usually explained by dust obscuration (Bouvier et al. 1995). When opaque material obscures the star, we see both reddened and scattered photons, but in the deepest minima the obscuration is so important that we mostly see scattered photons. If this opaque material is located in the circumstellar disk, this implies that the system inclination cannot be too small for obscuration to occur. The fact that inverse P Cygni profiles are often observed in GQ Lup A (Batalha et al. 2001) also favors a higher inclination for the system, since the redshifted absorptions are thought to come from accretion shock photons absorbed by high-velocity material in the funnel flow, so the accretion shock must be seen through the funnel in our line of sight (Muzerolle et al. 1998). The above results favor the inclination proposed in this work, $51^{\circ} \pm 13^{\circ}$, instead of that obtained by Broeg et al. (2007), $27^{\circ} \pm 5^{\circ}$, for GQ Lup A. The different inclination values are due to different periods and radii calculated for the system in both works. Unfortunately, precise CTTS periods are often hard to be obtained, and it is common to see different periods assigned to the same star, which have been calculated in different epochs (Bouvier et al. 1995). To see the influence of the period in the inclination value, using our proposed stellar radius and the Broeg et al. (2007) calculated period, we obtained $39^{\circ} \pm 12^{\circ}$, which agrees within the errors with our estimated system inclination (see Sect. 3.4.4).

Depending on a combination of the rotation ( $i$ ) and magnetic $(\beta)$ axis inclinations, the accretion ring can be totally or partially occulted as the system rotates (Mahdavi \& Kenyon 1998). Since 
the veiling values in this system never go to zero at all wavelengths, we assume we do not have a total occultation of the hot ring. Following the results of Mahdavi \& Kenyon (1998) (see their Fig. 6, right panel), for a star-disk system inclination of $i \approx 51^{\circ}$, the accretion region is never totally occulted if $\beta \leq 57^{\circ}$. We can also use their Fig. 9 to estimate a lower limit for $\beta$. In order to do so we need to input the veiling variability and the corresponding magnitude variability of GQ Lup A. The veiling in the $B$ band of our data varies from 0 to 1.5 (Sect. 3.1), but it can reach values of up to 4 in that spectral region (BLB), while the available $B$ band light curve presents variations of up to 2 mag. Mahdavi \& Kenyon (1998) showed that in order to obtain a 2 mag variation in the light curve $(\Delta m=2 \mathrm{mag})$ due to veiling alone, the maximum veiling value has to be approximately 10, independently of the values of $i$ and $\beta$. Since veiling values of 10 have never been measured in the $B$ band region of GQ Lup A, we conclude that veiling alone cannot be the cause of the 2 mag photometric variability. The $B$ band light curve of GQ Lup A is composed of a smooth variation with amplitude of $\Delta m=1$ mag (Fig. 5) that we tentatively attribute to veiling changes and a few points that fall out of this variability range that could be due to occultations by dust in the inner disk, like in AA Tau (Bouvier et al. 2003, 2007). If this speculation is correct, following Mahdavi \& Kenyon (1998) (their Fig. 9) with $\Delta m=1$ and $v=4(\mathrm{BLB})$ this implies that $\beta>45^{\circ}$ for $i=45^{\circ}$ and $\beta>35^{\circ}$ for $i=60^{\circ}$. If we consider $v=1.5$ (Sect. 3.1) then $\beta>55^{\circ}$ for $i=45^{\circ}$ and $\beta>45^{\circ}$ for $i=60^{\circ}$. Putting all the restrictions to $\beta$ together, we have $35^{\circ}<\beta<57^{\circ}$, considering that the 1 mag smooth variability observed in the $B$ band is only due to hot spots. However, cold magnetic spots can also contribute to the light curve variability of young stars as much as $0.8 \mathrm{mag}$ in $V$, as is often observed in the light curve of WTTSs, such as V410 Tau (Ménard \& Bertout 1999). The observed light curve modulation in CTTSs could then be due to a combination of dust occultation, cold and hot spots contributions and with the present data we are not able to determine the importance of each effect to the light curve variability.

\section{Conclusions}

We have obtained the main physical parameters of the CTTS GQ Lup A and tested some predictions of magnetospheric accretion models. The veiling was found to be periodic at $10.7 \pm 1.6$ days, which is consistent with the period obtained from the $B$ band photometry available in the literature $(10.43 \pm 0.12$ days $)$ and that we assumed to be the rotational period of the star. The periodic veiling variations can be explained by a CTTS with a magnetic field inclined with respect to the rotation axis. The star presents strong emission lines that vary substantially and are all strongly correlated with each other, but none were found to be correlated with veiling. This suggests that the main emission lines share some common formation region but they are not strongly influenced by the processes at work at the accretion shock region. Comparing the emission line profiles of $\mathrm{NaD}$ and $\mathrm{H} \beta$ with magnetospheric accretion profiles we estimate the mass accretion rate of the system. We determined the GQ Lup A photospheric flux and obtained the stellar radius and luminosity. Using standard evolutionary models, we also obtained the stellar mass and age. Our results are consistent with available published values for the studied parameters.

Acknowledgements. This research is based on data collected on the ESO $1.52 \mathrm{~m}$ telescope at La Silla, partly run by the Observatório Nacional (Brazil) as a result of the Brazilian-ESO agreement. We would like to thank the Extragalactic Astronomy Group of the Observatório Nacional (Brazil) for obtaining part of the data used in this paper. ESD acknowledges support from CAPES. SHPA acknowledges support from $\mathrm{CNPq}$ and Fapemig.

\section{References}

Alencar, S. H. P., \& Basri, G. 2000, AJ, 119, 1881

Appenzeller, I., Mundt, R., \& Wolf, B. 1978, A\&A, 63, 289

Appenzeller, I., Krautter, J., Mundt, R., Chavarria, C., \& Wolf, B. 1980, A\&A, 90,184

Baraffe, I., Chabrier, G., Allard, F., \& Hauschildt, P. H. 1998, A\&A, 337, 403

Batalha, C., Lopes, D. F., \& Batalha, N. M. 2001, ApJ, 548, 377

Batalha, C., Batalha, N. M., Alencar, S. H. P., Lopes, D. F., \& Duarte, E. S. 2002, ApJ, 580, 343

Bertout, C., Wolf, B., Carrasco, L., \& Mundt, R. 1982, A\&AS, 47, 419

Bertout, C., Basri, G., \& Bouvier, J. 1988, ApJ, 330, 350

Bouvier, J., Covino, E., Kovo, O., et al. 1995, A\&A, 299, 89

Bouvier, J., Grankin, K. N;, Alencar, S. H. P et al. 2003, A\&A, 409, 169

Bouvier, J., et al. 2007, A\&A, in press

Le Borgne, J.-F., Bruzual, G., Pelló, R., et al. 2003, A\&A, 402, 433

Broeg, C., Schmidt, T. O. B., Guenther, E., et al. M. 2007, A\&A, 468, 1039

Calvet, N., \& Gullbring, E. 1998, ApJ, 509, 802

Cayrel de Strobel, G., Soubiran, C., \& Ralite, N. 2001, A\&A, 373, 159

Covino, E., Terranegra, L., Franchini, M., Chavarria, K., \& Stalio, R. 1992, A\&A, 94, 273

Crawford, I. A. 2000, MNRAS, 317, 996

D'Antona, F., \& Mazzitelli, I. 1997, Mem. Soc. Astron. It., 68, 807

Foster, G. 1995, AJ, 109, 1889

Franco, G. A. P. 2000, MNRAS, 331, 474

Guenther, E. W., Neuhäuser, R., Wuchterl, G. P. H. 2005, Astron. Nachr., 326, 958

Gullbring, E., Hartmann, L., Briceno, C., \& Calvet, N. 1998, ApJ, 492, 323

Gullbring, E., Calvet, N., Muzerolle, J., \& Hartmann, L. 2000, ApJ, 544, 927

Hartmann, L., Hewett, R., \& Calvet, N. 1994, ApJ, 426, 669

Herbig, G. H., \& Bell, K. R. 1988, Third Catalog of Emission Line Stars of the Orion Population Lick Observatory Bulletin 1111, Santa Cruz

Herbst, W., unpublished Las Campanas data available at

http: //wWw . astro. wesleyan. edu/pub/ttauri/

Herbst, W., Bailer-Jones, C. A. L., Mundt, R., Meisenheimer, K., \&

Wackermann, R. 2002, A\&A, 396, 513

Horne, J.H., \& Baliunas, S.L. 1986, ApJ, 302, 757

Hughes, J., Hartigan, P., Krautter, J., \& Kelemen, J. 1994, AJ, 108, 1071

Janson, M., Brandner, W., Henning, T., \& Zinnecker, H. 2006, A\&A, 453, 609

Johns-Krull, C. M., \& Basri, G. 1997, ApJ, 474, 433

Kurosawa, R., Harries, T. J., \& Symington, N. H. 2006, MNRAS, 370, 580

Landin, N. R., Ventura, P., D’Antona, F., Mendes, L. T. S., \& Vaz, L. P. R. 2006, A\&A, 456, 269

Mahdavi, A., \& Kenyon, S. J. 1998, ApJ, 497, 342

Mathieu, R. D., Baraffe, I., Simon, M., Stassun, K. G., \& White, R. 2007, Protostars and Planets V, 411

McElwain, M. W., et al. 2007, ApJ, accepted

Ménard, F., \& Bertout, C. 1999, NATO ASIC Proc. 540: The Origin of Stars and Planetary Systems, 341

Mundt, R., \& Bastian, U. 1980, A\&A, 39, 245

Muzerolle J., Hartmann L., \& Calvet N. 1998, AJ, 116, 455

Muzerolle J., Hartmann L., \& Calvet N. 2001, ApJ, 550, 944

Neuhäuser, R., Guenther, E. W., Wuchterl, G. 2005, A\&A, 435, L13

Neuhaeuser, R., Mugrauer, M., Seifahrt, A., Schmidt, T., \& Vogt, N. 2008, [arXiv:0801.2287]

Perryman, M. A. C., et al. 1997, A\&A, 323, L49

Petrov, P. P., Gahm, G. F., Gameiro, J. F. 2001, A\&A, 369, 993

Pojmanski G. 2002, Acta Astron., 52, 397

Romanova, M. M., Ustyugova, G. V., Koldoba, A. V., \& Lovelace, R. V. E. 2004, ApJ, 610, 920

Scargle, J. D. 1982, ApJ, 263, 835

Schwartz R., \& Noah P., 1978, AJ, 83, 785

Shu F., Najita J., Ostriker E., et al. 1994, ApJ, 429, 781

Siess, L. 2001, From Darkness to Light: Origin and Evolution of Young Stellar Clusters, 243, 581

Siess, L., Dufour, E., \& Forestini, M. 2000, A\&A, 358, 593

Schmidt-Kaler, T. H. 1982, Physical Parameters of Stars. Landolt-Bornstein New Series. v.2, ed. K. Schaifers \& H. H.Voigt (Springer)

Torres, C. A. O., Quast, G. R., \& da Silva, L. 2006, A\&A, 460, 695

Vanmunster, A., unpublished Light Curve and Period Analysis Software available at http: //WWW . peranso.com/ 